\title{
Pessoas com deficiência no cenário rural: concepção dos Agentes Comunitários de Saúde
}

Disabled persons in the rural setting: conception of Community Health Workers

Personas con discapacidad en el entorno rural: concepción de Agentes Comunitarios de Salud

\section{Daiane Zeni', Marta Cocco da Costa ${ }^{\mathrm{II}}$, Fabiana Aparecida Rockemback ${ }^{\mathrm{III}}$, Deborah Tainara Schenkeliv ${ }^{\mathrm{IV}}$ Jaqueline Arboit ${ }^{\mathrm{v}}$, Fernanda Honnef ${ }^{\mathrm{vI}}$}

Resumo: Objetivo: descrever as concepções dos Agentes Comunitários de Saúde sobre as pessoas com deficiência que vivem em contexto rural. Método: pesquisa descritivo-exploratória de abordagem qualitativa, realizada em quatro municípios da região noroeste do Rio Grande do Sul, Brasil. Participaram treze Agentes Comunitários de Saúde. A coleta de dados ocorreu entre janeiro e fevereiro de 2018 por meio de entrevistas semiestruturadas, analisadas pela modalidade temática. Resultados: as concepções dos profissionais estão ancoradas especialmente nas palavras "dificuldade”, "atenção”, “cuidado", “desprezo" e "acesso”. Ademais, desvelam o conhecimento insuficiente acerca das pessoas com deficiência residentes no cenário rural, ao desconhecerem os diferentes tipos de deficiências existentes ou apontarem condições que não se enquadram em sua conceituação. Conclusão: evidenciou-se a necessidade premente de instrumentalização destes profissionais de saúde sobre o tema, a fim de proporcionar uma atenção à saúde com base na integralidade e equidade.

Descritores: Pessoas com deficiência; Zona rural; Atenção primária à saúde; Agentes comunitários de saúde; Saúde da população rural

Abstract: Objective: to describe the conceptions of Community Health Workers about disabled persons who live in rural contexts. Method: descriptive-exploratory research, with a qualitative approach, performed in four municipalities in the northwest region of Rio Grande do Sul, Brazil. It had the participation of thirteen Community Health Workers. Data collection took place between January and February 2018 through semi-structured interviews,

\footnotetext{
${ }^{\text {I }}$ Enfermeira. Residente do Programa de Residência Multiprofissional em Saúde/UFFS. Marau, RS, Brasil. E-mail: daiannezeni@hotmail.com ORCID: https://orcid.org/0000-0002-1299-2110

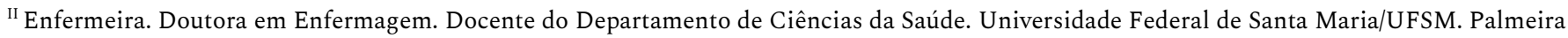
das Missões, RS, Brasil. E-mail: marta.c.c@ufsm.br ORCID: https://orcid.org/0000-0002-9204-3213

III Graduanda em Enfermagem. Universidade Federal de Santa Maria/UFSM. Palmeira das Missões, RS, Brasil. E-mail: rockembackfabiana@gmail.com ORCID: https://orcid.org/0000-0003-1786-1539

IV Graduanda em Enfermagem, Universidade Federal de Santa Maria/UFSM. Palmeira das Missões, RS, Brasil. E-mail: deborahtschenkel@gmail.com ORCID: https://orcid.org/0000-0002-9459-6110

v Enfermeira. Doutora em Enfermagem. Docente substituta do Departamento de Enfermagem. Universidade Federal de Santa Maria/UFSM. Santa Maria, RS, Brasil. E-mail: jaqueline.arboit@hotmail.com ORCID: https://orcid.org/0000-0002-6610-5900

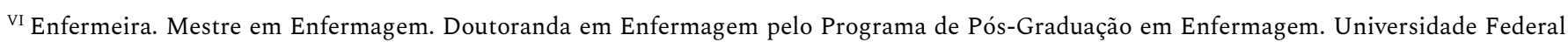
de Santa Maria/UFSM. Santa Maria, RS, Brasil. E-mail: fernandah.honnef@gmail.com ORCID: https://orcid.org/0000-0002-1866-1611
} 
Pessoas com deficiência no cenário rural: concepção dos Agentes Comunitários de Saúde| 2

analyzed by thematic modality. Results: the conceptions of these professionals are especially anchored in the words "difficulty", "attention", "care", "contempt" and "access". Moreover, they reveal the poor knowledge about disabled persons living in the rural setting, by ignoring the different types of disabilities or pointing out conditions that do not fit their conception. Conclusion: we should highlight the urgent need for instrumentalization of these health professionals on the theme, in order to provide health care based on integrality and equity.

Descriptors: Disabled persons; Rural areas; Primary health care; Community health workers; Rural health

Resumen: Objetivo: describir las concepciones de Agentes Comunitarios de Salud sobre las personas con discapacidad que viven en un contexto rural. Método: investigación descriptiva-exploratoria, con enfoque cualitativo, realizada en cuatro ayuntamientos de la región noroeste de Rio Grande do Sul, Brasil. Participaron trece Agentes Comunitarios de Salud. La recolección de datos tuvo lugar entre enero y febrero de 2018 mediante entrevistas semiestructuradas, analizadas por modalidad temática. Resultados: las concepciones de los profesionales están ancladas especialmente en las palabras "dificultad", "atención", "cuidado", "desprecio" y "acceso". Además, revelan el conocimiento insuficiente sobre las personas con discapacidad que viven en el entorno rural, al ignorar los diferentes tipos de discapacidad que existen o al señalar condiciones que no se ajustan a su concepción. Conclusión: se evidenció la necesidad urgente de instrumentalizar a estos profesionales de la salud sobre el tema, con miras a brindar una atención médica basada en la integralidad y la equidad.

Descriptores: Personas con discapacidad; Medio rural; Atención primaria de salud; Agentes comunitarios de salud; Salud rural

\section{Introdução}

Historicamente, no Brasil, muitos movimentos têm buscado a atenção integral à pessoa com deficiência, o que converge com os princípios do Sistema Único de Saúde (SUS). ${ }^{1}$ O conceito relativo à deficiência tem evoluído mundialmente, especialmente após os anos de 1960, quando passou a ser concebida como reflexo da estreita relação entre as limitações que as pessoas com deficiência experimentam, a estrutura do meio ambiente e as atitudes da comunidade. $^{2}$

Conforme o Censo de 2010, 45,6 milhões de pessoas, ou seja, 23,9\% do total da população brasileira possuem algum tipo de deficiência, seja visual, auditiva, motora, mental ou intelectual. Desta população, 7.132 .347 pessoas residem em áreas rurais. Entre os estados brasileiros, o Rio Grande do Sul apresenta umas das maiores taxas de pessoas com deficiência do país, correspondente a $22,5 \%$ da população deste estado. ${ }^{3}$ 
Em nível mundial, reconhece-se que estas pessoas enfrentam dificuldades generalizadas para acessar serviços de saúde, de educação e assistência social, incluindo moradia e transporte. Tais dificuldades as tornam mais vulneráveis a apresentar problemas de saúde em comparação com pessoas sem deficiência. Além disso, são relatadas entre as pessoas com deficiência maiores taxas de pobreza, de desemprego e de exclusão social. ${ }^{4}$

Embora no espaço urbano tais dificuldades de acesso a serviços e exclusão estejam presentes, estas se agravam ao se considerar o cenário rural. ${ }^{5}$ As dificuldades de acesso dessa população, especificamente em relação aos serviços de saúde são mostradas por estudos internacionais e nacionais. ${ }^{6-10}$ Nesta perspectiva, são descritas barreiras geográficas, como a distância até os serviços de saúde, as condições de terrenos e estradas, e as barreiras de transporte, como a ausência de transporte público e transporte privado de alto custo. ${ }^{7-8,10}$ Assim como barreiras organizacionais, a exemplo da falta de recursos e demora no atendimento das pessoas com deficiência; barreiras relacionadas ao comportamento dos profissionais, como o estigma ${ }^{7,9-10}$ e dificuldades de comunicação. ${ }^{10}$ Tais limitações que o cenário rural apresenta somadas às características físicas e/ou intelectuais das pessoas com deficiência acabam conferindo-lhes invisibilidade, ${ }^{5}$ limitando suas possibilidades.

Nesta direção, no intuito de transpor essas dificuldades e promover a articulação entre as pessoas com deficiência que residem no cenário rural e os serviços de saúde, tem-se a Atenção Primária a Saúde (APS). Esta consiste em um conjunto de ações que incluem a promoção, prevenção, proteção, diagnóstico, tratamento e reabilitação da saúde, desenvolvidas por uma equipe multiprofissional em território definido e direcionadas a indivíduos, famílias e coletividades. ${ }^{11}$

No âmbito da APS, dentre os profissionais que desenvolvem suas ações no cenário rural, destaca-se o Agente Comunitário de Saúde (ACS), o qual faz parte da equipe da Estratégia de Saúde da Família (ESF). O ACS atua como elo de aproximação das ações de saúde com a 
Pessoas com deficiência no cenário rural: concepção dos Agentes Comunitários de Saúde| 4

população, especialmente em áreas de difícil acesso e com grupos marginalizados, ${ }^{12}$ como as pessoas com deficiência. Assim, o papel do ACS se torna ainda mais relevante no cenário rural, consideradas as suas singularidades. Este profissional, dentre outras atribuições, realiza o cadastramento das famílias de sua microárea de abrangência, orienta as famílias quanto aos serviços de saúde e desenvolve intervenções que visam à promoção da saúde e prevenção de agravos mediante visitas domiciliares, como a verificação da situação vacinal. ${ }^{13-14}$

Frente à problemática exposta, que evidencia a complexidade que envolve o acesso à saúde das pessoas com deficiência que residem em cenários rurais, há necessidade de qualificação das ações implantadas, considerando as vulnerabilidades vivenciadas por esta população. ${ }^{6}$ Assim, buscam-se, com o estudo, subsídios para o desenho de políticas públicas e qualificação das práticas de atenção à saúde com vistas à promoção do cuidado às pessoas com deficiência residentes no contexto rural, partindo da visão dos ACS. Para tanto, tem-se como objetivo descrever as concepções dos ACS sobre as pessoas com deficiência que vivem em contexto rural.

\section{Método}

Pesquisa descritivo-exploratória de abordagem qualitativa, realizada em quatro municípios pertencentes à $15^{\mathrm{a}}$ Coordenadoria Regional de Saúde (CRS), localizada na região noroeste do Estado do Rio Grande do Sul. A $15^{\mathrm{a}}$ CRS é composta por 26 municípios, com uma população de 166.764 habitantes. ${ }^{15}$

Para elencar o cenário do estudo, buscaram-se os municípios da referida região de saúde que possuíssem mais de $70 \%$ de seus residentes no meio rural e que fossem assistidos por ACS. Listaram-se quatro com tais características. Pontua-se que estes não dispunham de informação relativa ao número exato de pessoas com deficiência da sua população rural, apenas de que elas existiam, o que revela a sua invisibilidade. Ademais, nestes municípios não havia unidade de ESF localizada no cenário rural. Assim, os ACS eram os principais profissionais que 
desenvolviam as ações junto à população neste cenário, por meio das visitas domiciliares. Estas eram realizadas por outros profissionais apenas quando existiam demandas específicas e não havia possibilidade de o usuário se deslocar até a unidade da ESF.

Após aprovação dos gestores, os pesquisadores contataram os 15 ACS que desenvolviam suas atividades nas áreas rurais do cenário do estudo para convidá-los a participar. Este convite foi realizado de forma verbal pela pesquisadora responsável e uma auxiliar de pesquisa durante reuniões agendadas com os ACS em cada município. Utilizou-se como critério de inclusão estar atuando no serviço havia mais de seis meses; e como critério de exclusão estar em licença ou férias. Destes ACS, dois estavam em férias e os demais aceitaram participar do estudo. Assim, foram participantes deste estudo 13 ACS, cada um deles responsável por uma microárea no seu município. Do total de participantes por município, em três deles participaram três ACS e, em outro, quatro.

Para a geração dos dados, utilizou-se a entrevista semiestruturada e individual, empregando um roteiro com perguntas abertas relacionadas aos dados sociodemográficos e de formação dos ACS, as suas concepções sobre as pessoas com deficiência residentes em cenários rurais e seu cotidiano laboral na atenção a elas. Ao iniciar a entrevista, foi solicitado aos ACS que citassem palavras que os remetessem a pessoas com deficiência no meio rural. A evocação pelos participantes no início da entrevista teve como intuito subsidiar a construção de uma nuvem de palavras, que as agrupa e organiza de acordo a sua frequência. Nesta nuvem, as palavras são dispostas de forma aleatória, e as mais citadas ficam mais representativas. Isso significa que cada palavra tem seu tamanho regido pela relevância em determinado corpus textual. ${ }^{16}$ Esta nuvem foi construída a partir de um programa de acesso livre e gratuito on-line. Após este momento inicial, foi dada sequência, com as demais perguntas. A coleta de dados encerrou quando foi compreendida a lógica interna do objeto de estudo, a partir da reincidência e complementaridade das informações. ${ }^{17}$ 
Pessoas com deficiência no cenário rural: concepção dos Agentes Comunitários de Saúde| 6

Visando à privacidade dos participantes, as entrevistas foram previamente agendadas e realizadas no horário escolhido por cada ACS, em uma sala reservada nas unidades de ESF de cada município. Elas foram gravadas em gravador digital (áudio), tendo duração média de 30 minutos. A geração dos dados ocorreu nos meses de janeiro e fevereiro de 2018.

Após a realização das entrevistas, estas foram transcritas de modo literal em um programa editor de textos. Na sequência, o material empírico transcrito foi submetido à análise de conteúdo temática, composta por três etapas: a pré-análise, exploração do material, e tratamento dos resultados obtidos e interpretação. ${ }^{18} \mathrm{Na}$ pré-análise, fez-se a leitura flutuante e a constituição do corpus conforme objetivo proposto. $\mathrm{Na}$ exploração do material, realizou-se a codificação por meio das palavras ou frases semelhantes nos discursos, elaborando as categorias. No tratamento dos resultados e interpretação, pôde-se agrupar os discursos, visualizar as informações obtidas e relacioná-las com as evidências científicas referentes ao objeto do estudo. ${ }^{18}$ Desta análise, emergiram duas categorias temáticas: Concepções dos ACS sobre as pessoas com deficiência por meio da representatividade das palavras; e Invisibilidade das múltiplas deficiências no contexto rural na voz dos ACS.

O estudo seguiu as recomendações expressas por meio da Resolução nº. 466, de 12 de dezembro de 2012, do Conselho Nacional de Saúde. ${ }^{19} \mathrm{O}$ mesmo foi aprovado em 9 de agosto de 2017 pelo Comitê de Ética em Pesquisa da Universidade Federal de Santa Maria, com o Parecer $\mathrm{n}^{\circ}$ 2.208.566. Os ACS foram informados acerca dos objetivos e método por meio da leitura e explicação do Termo de Consentimento Livre e Esclarecido e aqueles que concordaram em participar o assinaram em duas vias, ficando uma com o participante e a outra com a pesquisadora. Para o seu anonimato, os depoimentos serão identificados pela sigla ACS seguida do número correspondente à ordem de realização da entrevista (ex.: ACS 1, ACS 2... ACS13). 
7 | Zeni D, Costa MC, Rockemback FA, Schenkel, Arboit J, Fernanda F

\section{Resultados}

Do total de participantes do estudo, 10 eram do sexo feminino e três, do sexo masculino. A média de idade foi de 36,9 anos. Quanto à escolaridade, nove possuíam Ensino Médio completo; três, Ensino Superior completo; e um, Ensino Fundamental completo. O tempo de atuação como ACS variou de seis meses a 16 anos, com média de 6,6 anos.

Concepções dos ACS sobre as pessoas com deficiência por meio da representatividade das palavras

Na Figura 1, é apresentada a nuvem de palavras a partir da análise das evocações dos ACS que remetiam às pessoas com deficiência em cenários rurais. No total, foram evocadas 65 palavras. As mais citadas durante as entrevistas e que se assemelhavam quanto ao seu significado foram: dificuldade e/ou dificuldades (12,3\%); atenção e cuidado (9,2\%); desprezo, dó, carente e carinho (7,7\%); e acesso (4,6\%).

Figura 1- Representação "nuvem de palavras" por meio da consulta pela frequência de palavras. Palmeira das Missões/RS, Brasil, 2019

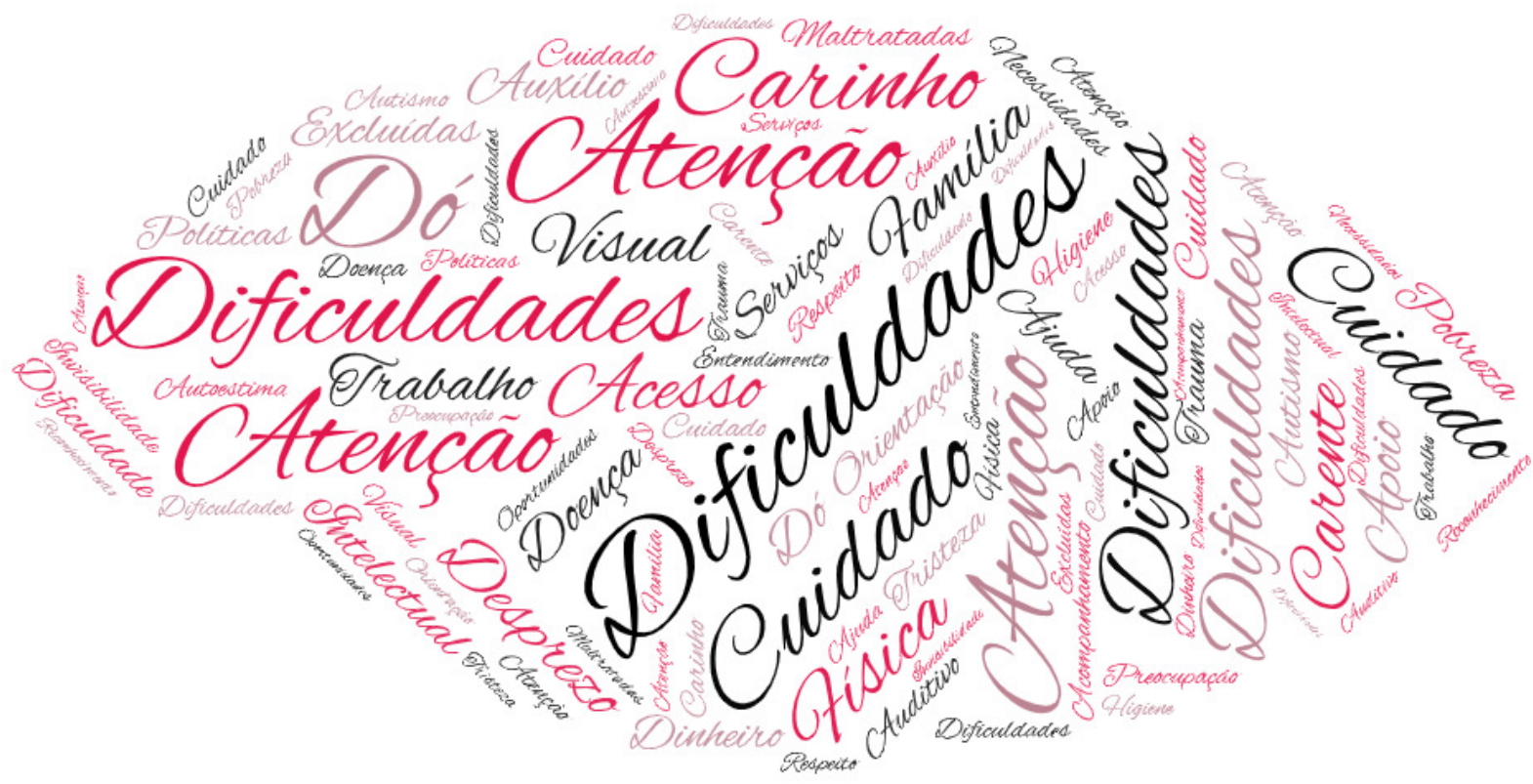


Pessoas com deficiência no cenário rural: concepção dos Agentes Comunitários de Saúde| 8

Os depoimentos permitem ilustrar as palavras que foram citadas com maior frequência e que compuseram a nuvem.

Deficiência [...] eu acho que é uma pessoa que precisa de mais cuidado, mais atenção. (ACS 1)

Eu associo a deficiência à dificuldade para vim até o posto de saúde, no caso [...] caso um dia precise vir aqui tomar uma vacina, é difícil. (ACS 5)

Eu acho que deficiência significa, principalmente, uma pessoa que precisa de mais carinho e mais atenção [...] porque hoje na sociedade está sendo bastante desprezado[...]. (ACS7)

Ele [se referindo a um jovem com deficiência física] é uma pessoa que quer alcançar algo melhor [...] ele já procurou muito emprego e não conseguiu ainda [...] não é fácil o acesso ao campo de trabalho [...] é uma dificuldade da pessoa com deficiência. (ACS8)

Deficiência significa uma pessoa especial [...] que tem que ter um cuidado especial. (ACS 10)

Uma pessoa com dificuldade [...] que às vezes as outras pessoas não aceitam, deixam mais excluídas. (ACS 12)

Nesta categoria, foi possível evidenciar que os participantes representaram as pessoas com deficiência a partir das palavras: dificuldade, atenção, cuidado, desprezo, dó, carente, e carinho. Elas remetem ao cotidiano destas pessoas, permeado por demandas, dificuldades de acesso a serviços e espaço no mercado de trabalho, bem como pela exclusão social.

\section{Invisibilidade das múltiplas deficiências no contexto rural na voz dos ACS}

Nos depoimentos, os ACS citaram apenas alguns tipos de deficiência, desconhecendo a totalidade das deficiências existentes. Ainda, apontaram condições que não se enquadram na conceituação de deficiência. 
9 |Zeni D, Costa MC, Rockemback FA, Schenkel, Arboit J, Fernanda F

Para mim, deficiência na minha área [...] tem deficiência visual, deficiência intelectual, auditiva, tem física. (ACS 3)

Deficiência, para mim, significa qualquer tipo de perda ou anormalidade que limitam a função física ou mental. (ACS 4)

Muitas pessoas com problemas depressivos [...] eu acho que a depressão é uma deficiência. (ACS 5)

Tem a deficiência física [...] a deficiência cognitiva. (ACS6)

Na minha área tenho a deficiência física. (ACS 10)

A pessoa com deficiência tem dificuldade de se locomover [...] problema de visão. (ACS 12)

Os depoimentos que seguem revelam o desconhecimento dos ACS em relação às pessoas com deficiência.

Não tenho muito conhecimento. (ACS 4)

A gente tem pouca informação sobre [referindo-se às pessoas com deficiência]. (ACS5)

A gente tinha que ter uma capacitação melhor para trabalhar com essas pessoas [...] a gente não tem informação sobre o que é [...] nem preparo. (ACS 13)

Esta categoria permite identificar a invisibilidade e o conhecimento insuficiente acerca das pessoas com deficiência residentes no cenário rural pelos ACS, evidenciando a pouca qualificação que possuíam para assistir a esta população. 
Pessoas com deficiência no cenário rural: concepção dos Agentes Comunitários de Saúde| 10

\section{Discussão}

Com relação à primeira categoria temática, as palavras mais mencionadas pelos ACS durante as entrevistas foram associadas com as dificuldades que as pessoas com deficiência residentes em cenários rurais enfrentam. Outras concepções foram reveladas a partir da evocação de “atenção” e "cuidado”, seguidas de “desprezo”, “dó”, “carente” e "carinho”. Os ACS também tinham suas concepções imbuídas pelos aspectos do acesso das pessoas com deficiência residentes em cenário rural aos serviços de saúde e ao mundo do trabalho.

As concepções representadas pela palavra "dificuldade" relacionam-se com as dificuldades encontradas no cotidiano de vida dessa população presentes no contexto familiar, em espaços públicos e no acesso a serviços. As pessoas com deficiência apresentam relações sociais limitadas ou inexistentes, restringindo-se geralmente à esfera familiar. ${ }^{20}$ Com base nestas concepções de dificuldade, desvela-se a necessidade iminente de ampliação do acesso às políticas públicas baseadas nas particularidades da vida de pessoas com deficiência nos cenários rurais. ${ }^{6}$

A representação das palavras “atenção" e “cuidado” carregam concepções acerca da necessidade de promover uma atenção fundamentada na integralidade, considerando as necessidades singulares e complexas das pessoas com deficiência. Sob este aspecto, a literatura evidencia que a atenção à saúde das pessoas com deficiência que vivem no cenário rural por profissionais de saúde da APS ainda se encontra centrada no modelo biomédico, desconsiderando os aspectos socioculturais dos usuários e norteando-se pelo modelo baseado nas características biológico-corporais, influenciando diretamente na resolutividade do serviço. ${ }^{6}$

Outra concepção dos ACS é desvelada a partir das palavras “desprezo”, “dó”, “carente” e “carinho”, remetendo à sensibilização destes profissionais no que se refere às situações de vida dessas pessoas. Embora essa sensibilização seja positiva, estudo apontou que elas não se sentem confortáveis tendo sua condição associada à caridade e piedade, desejando ser vistas como pessoas capazes. $^{21}$ 
O desprezo que um dos ACS relatou representa a estigmatização vivenciada pelas pessoas com deficiência, situação frequente em seus cotidianos. A literatura aponta que o estigma é uma importante barreira a estas pessoas, especialmente para aquela que reside no cenário rural. O medo e receio de sofrer preconceito ao saírem de suas residências atua como fator desfavorável ao seu acesso aos serviços de saúde, ${ }^{7}$ fazendo com que muitas deixem de buscar tais serviços. Neste cenário de estigmatização cotidiana, indivíduos com deficiência permanecem marginalizados pela sociedade, impossibilitados de exercer de forma plena seus direitos, ${ }^{22}$ sendo um deles o direito à saúde, garantido pela Constituição Federal brasileira.

Outra palavra expressa pelos participantes foi "carinho", o que permite depreender certa sensibilidade desses profissionais quanto às realidades em que as pessoas com deficiência que vivem no cenário rural estão inseridas. No entanto, para além de ser sensível frente a esta realidade, faz-se necessário transformá-la em práticas de atenção efetivas e que considerem esses indivíduos não apenas em sua dimensão biológica. Tais práticas podem envolver a inclusão social e promoção da sua autonomia e qualidade de vida por meio de grupos comunitários em integração a outros serviços da Rede de Cuidados à Pessoa com Deficiência.

A palavra “acesso" também foi evocada pelos ACS, estando relacionada às dificuldades de ir até o serviço de APS e de conquistar uma vaga no mercado de trabalho. Com relação aos serviços e cuidados de saúde, esta compreendia uma das principais preocupações quando se tratava da população rural, e principalmente quando possuía algum tipo de deficiência. Sobre tal aspecto, é relevante considerar que o conceito de acesso está intimamente relacionado ao de acessibilidade. A acessibilidade diz respeito à possibilidade que o usuário tem ou não de chegar ao serviço de saúde. O acesso, por sua vez, vincula-se à oferta deste serviço, permitindo o uso oportuno deste para atingir os melhores resultados de saúde possíveis. ${ }^{23}$

Nessa perspectiva, estudo desenvolvido em uma comunidade rural da África do Sul revelou que as pessoas com deficiência enfrentam barreiras significativamente maiores ao 
Pessoas com deficiência no cenário rural: concepção dos Agentes Comunitários de Saúde| 12

acesso aos cuidados de saúde em comparação com aquelas sem deficiência, aumentando conforme o seu tipo e grau. ${ }^{24}$ As barreiras enfrentadas se relacionam especialmente à distância geográfica em relação aos centros urbanos, falta de transporte e falta de dinheiro para pagar por este transporte, deixando as pessoas com deficiência e as suas famílias a vivenciar restrições quanto ao direito à saúde e recursos coletivos de atenção social, proteção e assistência. ${ }^{7,24} \mathrm{O}$ contexto apresentado vai de encontro a um dos pressupostos da Convenção Internacional sobre os Direitos das Pessoas com Deficiência e de seu Protocolo Facultativo no Brasil, o qual declara que elas têm direito ao gozo do mais alto padrão de saúde possível, ${ }^{25}$ o que só será viável a partir do seu acesso aos serviços de saúde.

No que tange ao acesso ao mercado de trabalho, historicamente estes indivíduos vêm pleiteando espaços de participação social, particularmente por meio do acesso à educação e ao trabalho. ${ }^{20}$ Embora a Lei Brasileira de Inclusão da Pessoa com Deficiência (Lei no 13.146 de 6 de julho de 2015) busque assegurar o seu direito à qualificação profissional, ao acesso e permanência no trabalho, bem como ao incentivo ao empreendedorismo e ao trabalho autônomo, ${ }^{26}$ existem barreiras físicas e atitudinais a serem superadas para que essa lei se efetive. ${ }^{20}$ Isso fica explícito a partir dos resultados de estudo brasileiro que revelou que os principais desafios para a inclusão das pessoas com deficiência no mercado de trabalho compreendem, dentre outros, a sua baixa qualificação, falta de acessibilidade, preconceito e o despreparo das empresas. ${ }^{27}$

A segunda categoria temática aborda a invisibilidade dos diferentes tipos de deficiência pela maioria dos ACS, que as limitam às esferas física e mental, desconsiderando as demais, quais sejam, a motora, auditiva e visual. Além disso, um dos participantes citou a depressão como um tipo de deficiência. No entanto, se trata de uma doença/transtorno mental. Tais concepções restritas e por vezes equivocadas pautam-se no conhecimento insuficiente destes 
profissionais em relação ao tema, o que é revelado também quando citaram informações e capacitações escassas sobre a deficiência.

As dificuldades dos ACS podem estar relacionadas às poucas vivências e/ou experiências e, principalmente, à formação e qualificações precárias. Sabe-se que muitas capacitações são realizadas de modo genérico, desconsiderando singularidades relacionadas aos cenários territoriais e populações. Para que as capacitações tenham êxito, devem partir da análise das necessidades dos ACS e estarem articuladas e em consonância com os objetivos e contexto de cada sistema de saúde, como prevê diretriz internacional. ${ }^{28}$

O conhecimento insuficiente dos ACS influencia diretamente na resolutividade das necessidades de saúde desses indivíduos. Tal situação também é apontada por estudo desenvolvido com profissionais de saúde em uma área rural do sul de Malawi, segundo o qual a maioria deles concebia a deficiência como uma doença, pautados no modelo médico de deficiência, resultando no atendimento fragmentado das necessidades de saúde dessas pessoas. ${ }^{29}$

Estudo que objetivou avaliar a confiabilidade das informações sobre pessoas com deficiência coletadas por ACS em serviços da APS, em duas cidades brasileiras, também identificou dificuldades relacionadas ao seu conhecimento. Isso porque os dados de prevalência de pessoas com deficiência registrados por estes profissionais revelaram uma subnotificação de aproximadamente $50 \%$, em comparação aos dados coletados pelos pesquisadores. ${ }^{30}$

Reitera-se que, sem a compreensão adequada do que é a deficiência e quais seus tipos, dificilmente os ACS conseguirão identificar as demandas das pessoas com deficiência e desenvolver ações para atendê-las. No cenário rural, isto se torna ainda mais grave, visto que estes profissionais são a base da comunicação entre as pessoas com deficiência e o serviço de saúde, especificamente da APS.

\section{Conclusão}


Pessoas com deficiência no cenário rural: concepção dos Agentes Comunitários de Saúde| 14

As concepções dos ACS estão ancoradas especialmente nas palavras "dificuldade”, “atenção”, “cuidado”, “desprezo” e “acesso”. Ademais, suas concepções desvelam a invisibilidade e o conhecimento insuficiente acerca das pessoas com deficiência residentes no cenário rural, ao desconhecerem os diferentes tipos de deficiências existentes ou apontar condições que não se enquadram na sua conceituação.

Por meio dos resultados foi possível evidenciar e reforçar o cenário de invisibilidade das pessoas com deficiência que residem em contexto rural. Isto acaba influenciando nas ações de cuidado direcionadas a esta população, visto que os ACS constituem a interlocução entre ela e a APS.

O estudo em tela apresentou limitações relacionadas à escassez de produções científicas contemplando a temática da pessoa com deficiência e o meio rural e a pequena amostra de um contexto específico. Assim, para a generalização dos resultados, este último aspecto deve ser considerado.

Apesar de tais limitações, apresenta contribuições ao abordar as concepções dos ACS acerca das pessoas com deficiência no cenário rural. A partir dessas concepções evidenciou-se a necessidade premente de instrumentalização destes profissionais de saúde sobre o tema, a fim de proporcionar uma atenção à saúde com base na integralidade e equidade, princípios do SUS. Para tanto, tendo em vista a complexidade do tema, o papel da Enfermagem é fundamental na supervisão e capacitação dos ACS, em conjunto com a equipe multiprofissional.

\section{Referências}

1. Campos MF, Souza LAP, Mendes VLF. A rede de cuidados do Sistema Único de Saúde à saúde das pessoas com deficiência. Interface Comun Saúde Educ. 2015;19(52):207-10. doi: 10.1590/180757622014.0078

2. Ministério da Saúde (BR), Secretaria de Atenção à Saúde, Departamento de Ações Programáticas Estratégicas. Política nacional de saúde da pessoa com deficiência [Internet]. Brasília (DF): Ministério da 
Saúde; 2010 [acesso em 2019 fev 20]. Disponível em: https://bvsms.saude.gov.br/bvs/publicacoes/politica_nacional_pessoa_com_deficiencia.pdf

3. Instituto Brasileiro de Geografia e Estatística (IBGE). Censo demográfico 2010: características gerais da população, religião e pessoas com deficiência [Internet]. Rio de Janeiro (RJ); 2010 [acesso em 2019 fev 19]. Disponível em: https://biblioteca.ibge.gov.br/visualizacao/periodicos/94/cd_2010_religiao_deficiencia.pdf

4. World Health Organization (WHO). WHO global disability action plan 2014-2021: better health for all people with disability [Internet]. Geneva: WHO; 2015 [cited 2019 Feb 22]. Available from: https://apps.who.int/iris/bitstream/handle/10665/199544/9789241509619_eng.pdf;jsessionid=D408684546F B90724F74F3F2CB948394? sequence=1

5. Tonini H, Lopes MJM. Desenvolvimento, cuidado e vulnerabilidade: pessoas com deficiência em áreas rurais do RS. Rev Interdiscip [Internet]. 2017 [acesso em 2019 jun 14];10(3):110-24. Disponível em: https://revistainterdisciplinar.uninovafapi.edu.br/index.php/revinter/article/view/1318

6. Ursine BL, Pereira EL, Carneiro FF. Saúde da pessoa com deficiência que vive no campo: o que dizem os trabalhadores da Atenção Básica? Interface Comun Saúde Educ. 2018;22(64):109-20. doi: 10.1590/180757622016.0666

7. Vergunst R, Swartz L, Mji G, MacLachlan M, Mannan H. 'You must carry your wheelchair' - barriers to accessing healthcare in a South African rural area. Glob Health Action. 2015;8:1. doi: $10.3402 /$ gha.v8.29003

8. Davidsson N, Södergård B. Access to healthcare among people with physical disabilities in rural Louisiana. Soc Work Public Health. 2016;31(3):188-95. doi: 10.1080/19371918.2015.1099496

9. Dassah E, Aldersey HM, McColl MA, Davison C. 'When I don't have money to buy the drugs, I just manage.' - Exploring the lived experience of persons with physical disabilities in accessing primary health care services in rural Ghana. Soc Sci Med. 2018;214:83-90. doi: 10.1016/j.socscimed.2018.08.023

10. Munthali AC, Swartz L, Mannan H, MacLachlan M, Chilimampunga C, Makupe C. This one will delay us": barriers to accessing health care services among persons with disabilities in Malawi. Disabil Rehabil. 2017;41(6):683-90. doi: 10.1080/09638288.2017.1404148

11. BRASIL. Ministério da Saúde. Portaria no 2.436, de 21 de setembro de 2017. Aprova a Política Nacional de Atenção Básica, estabelecendo a revisão de diretrizes para a organização da Atenção Básica, no âmbito do Sistema Único de Saúde (SUS). Diário Oficial da União: Edição 183, Seção 1, p. 68. Brasília, DF: Ministério da Saúde, 2017. Disponível em: http://www.in.gov.br/materia//asset_publisher/Kujrw0TZC2Mb/content/id/19308123/do1-2017-09-22-portaria-n-2-436-de-21-desetembro-de-2017-19308031. Acesso em: 14 jun. 2019.

12. McCollum R, Gomez W, Theobald S, Taegtmeyer M. How equitable are community health worker 
Pessoas com deficiência no cenário rural: concepção dos Agentes Comunitários de Saúde| 16

programmes and which programme features influence equity of community health worker services? A systematic review. BMC Public Health. 2016;16:419. doi: 10.1186/s12889-016-3043-8

13. Nunes CA, Aquino R, Medina MG, Vilasbôas ALQ, Pinto Júnior EP, Luz LA. Visitas domiciliares no Brasil: características da atividade basilar dos Agentes Comunitários de Saúde. Saúde Debate. 2018;42(N Esp 2):127-44. doi: 10.1590/0103-11042018s209

14. Barreto ICHC, Pessoa VM, Souza MFA, Nuto SAS, Freitas RWJF, Ribeiro KG, et al. Complexidade e potencialidade do trabalho dos Agentes Comunitários de Saúde no Brasil contemporâneo. Saúde Debate. 2018;42(N Esp 1):114-29. doi: 10.1590/0103-11042018s108

15. Secretaria Estadual de Saúde (Rio Grande do Sul), Grupo de Trabalho de Planejamento, Monitoramento e Avaliação. Plano Estadual de Saúde: 2016/2019 [Internet]. Porto Alegre (RS); 2016 [acesso em 2020 abr 20]. Disponível em: https://saude.rs.gov.br/upload/arquivos/201701/05153251-pes2016-2019-sesrs.pdf

16. Kami MTM, Larocca LM, Chaves MMN, Lowen IMV, Souza VMP, Goto DYN. Working in the street clinic: use of IRAMUTEQ software on the support of qualitative research. Esc Anna Nery Rev Enferm. 2016;20(3):e20160069. doi: 10.5935/1414-8145.20160069

17. Minayo MCS. Amostragem e saturação em pesquisa qualitativa: consensos e controvérsias. Rev Pesqui Qual [Internet]. 2017 [acesso em 2020 fev 09];5(7):1-12 Disponível em: https://editora.sepq.org.br/index.php/rpq/article/view/82/59

18. Minayo MCS. O desafio do conhecimento: pesquisa qualitativa em saúde. $14^{a}$ ed. São Paulo: Hucitec; 2014.

19. BRASIL. Ministério da Saúde. Resolução no 466, de 12 de dezembro de 2012. Diretrizes e normas regulamentadoras de pesquisas envolvendo seres humanos. Brasília, DF: Ministério da Saúde, 2013. Disponível em: http://bvsms.saude.gov.br/bvs/saudelegis/cns/2013/res0466_12_12_2012.html. Acesso em: 10 out. 2019.

20. Aoki M, Silva RM, Souto ACF, Oliver FC. Pessoas com deficiência e a construção de estratégias comunitárias para promover a participação no mundo do trabalho. Rev Bras Educ Espec. 2018;24(4): 51734. doi: $10.1590 / \mathrm{s} 1413-65382418000500004$

21. Visagie S, Swartz L. "There is nothing wrong with me": disability invisibility in a rural South African town. Disabil Rehabil. 2017;40(15):1799-807. doi: 10.1080/09638288.2017.1313909

22. Senjam SS, Singh A. Addressing the health needs of people with disabilities in India. Indian J Public Health. 2020;64(1):79-82. doi: 10.4103/ijph.IJPH_27_19

23. Starfield B. Atenção primária: equilíbrio entre necessidades de saúde, serviços e tecnologia. Brasília (DF): UNESCO, Ministério da Saúde; 2002. 
24. Vergunst R, Swartz L, Hem KG, Eide AH, Mannan H, MacLachlan M, et al. Access to health care for persons with disabilities in rural South Africa. BMC Health Serv Res. 2017;17:741. doi: 10.1186/s12913017-2674-5

25. BRASIL. Decreto no 6.949, de 25 de agosto de 2009. Promulga a Convenção Internacional sobre os Direitos das Pessoas com Deficiência e seu protocolo facultativo, assinados em Nova York, em 30 de março de 2007. Brasília, DF: Presidência da República, 2009. Disponível em: http://www.planalto.gov.br/ccivil_03/_ato2007-2010/2009/decreto/d6949.htm. Acesso em 14 jun. 2019.

26. BRASIL. Lei no 13.146, de 6 de julho de 2015. Institui a lei brasileira de inclusão da pessoa com deficiência (Estatuto da Pessoa com Deficiência). Brasília, DF: Presidência da República, 2015. Disponível em: http://www.planalto.gov.br/ccivil_03/_ato2015-2018/2015/lei/l13146.htm. Acesso em 14 jun. 2019.

27. Neves-Silva P, Prais FG, Silveira AM. The inclusion of disabled persons in the labor market in Belo Horizonte, Brazil: scenario and perspective. Ciênc Saúde Colet. 2015;20(8):2549-58. doi: 10.1590/141381232015208.17802014

28. Cometto G, Ford N, Pfaffman-Zambruni J, Akl EA, Lehmann U, McPake B, et al. Health policy and system support to optimize community health worker programmes: an abridged WHO guideline. Lancet Glob Health. 2018;6(12):e1397-404. doi: 10.1016/S2214-109X(18)30482-0

29. Braathen SH, Munthali A, Grut L. Explanatory models for disability: perspectives of health providers working in Malawi. Disabil Soc. 2015;30(9):1382-96. doi: 10.1080/09687599.2015.1099517

30. Lopes FRL, Monteiro KS, Figueiredo T, Wanderley TC, Pequeno TA, Lima S, et al. Reliability of information on people with disabilities gathered by community health workers in highly consanguineous communities of Northeastern Brazil. BMC Health Serv Res. 2017;17:317. doi: 10.1186/s12913-017-2267-3

\section{Autor correspondente}

Marta Cocco da Costa

E-mail: marta.c.c@ufsm.br

Endereço: Av. Independência, nº 3751,

Bairro Vista Alegre, Palmeira das Missões, RS, Brasil

CEP: $98300-000$

\section{Contribuições de Autoria}

\section{1 - Daiane Zeni}

Concepção e projeto, análise e interpretação dos dados, redação do artigo, revisão crítica relevante do conteúdo intelectual, aprovação final da versão a ser publicada. 


\section{2 - Marta Cocco da Costa}

Concepção e projeto, análise e interpretação dos dados, redação do artigo, revisão crítica relevante do conteúdo intelectual, aprovação final da versão a ser publicada.

\section{3 - Fabiana Aparecida Rockemback}

Análise e interpretação dos dados, redação do artigo, revisão crítica relevante do conteúdo intelectual, aprovação final da versão a ser publicada.

\section{4 - Deborah Tainara Schenkel}

Análise e interpretação dos dados, redação do artigo, revisão crítica relevante do conteúdo intelectual, aprovação final da versão a ser publicada

\section{5 - Jaqueline Arboit}

Análise e interpretação dos dados, redação do artigo, revisão crítica relevante do conteúdo intelectual, aprovação final da versão a ser publicada

\section{6 - Fernanda Honnef}

Análise e interpretação dos dados, redação do artigo, revisão crítica relevante do conteúdo intelectual, aprovação final da versão a ser publicada

\section{Como citar este artigo}

Zeni D, Costa MC, Rockemback FA, Schenkel, Arboit J, Fernanda F. Pessoas com deficiência no cenário rural: concepção dos Agentes Comunitários de Saúde. Rev. Enferm. UFSM. 2020 [Acesso em: Anos Mês Dia]; vol.10 e62: 1-18. DOI:https://doi.org/10.5902/2179769239660 